\title{
Medidas arancelarias
}

y no arancelarias

del comercio

internacional*

/ Tariff And

Non-Tariff Measures

In International

Trade

* Recibido: 18 de febrero de 2013. Aceptado: 29 de abril de 2013.

Tla-Melaua, revista de Ciencias Sociales. Facultad de Derecho y Ciencias Sociales. Benemérita Universidad Autónoma de Puebla, México / IssN: 1870-6916 / Nueva Época, Año 7 No 35, Octubre 2013-Marzo 2014, PP. 102-116. 
RESUMEN

$\mathrm{Al}$ hablar de las medidas arancelarias con las que se cuenta para la regulación del comercio internacional entre países, no se puede dejar de lado a las medidas no arancelarias. Estas medidas, en su momento consideradas peligrosas para el comercio internacional, razón que no es así, toda vez que en el último informe de la OMC 2012 acerca del Comercio y Políticas Publicas y el análisis de las medidas no arancelarias son más los beneficios para los gobiernos en cuanto al cumplimiento de sus políticas comerciales. El siguiente tema a desarrollar pretende brindar al lector un panorama más claro de las medidas arancelarias y no arancelarias en México y como ambas convergen en el comercio internacional. Primero, se analizará la definición de medida arancelaria, el concepto de medida no arancelaria a partir de lo definido por la OMC, sus características principales, los efectos y usos en el comercio nacional e internacional dentro del sistema multilateral.

\section{PALABRAS CLAVE}

Arancel, Medidas de Regulación, Comercio Internacional, Medidas No Arancelarias.
A B S TRACT

Speaking of tariff measures, which account for the regulation of international trade between countries, you cannot leave out non-tariff measures. These measures, at the time considered dangerous to international trade, because it is not so, since in the final WTO report 2012 on Trade and Public Policy, and the analyses of non-tariff measures are of more benefits to governments in meeting their trade policies. The next topic to be developed, is intended to provide the reader a clearer picture of the Mexico tariff measures, non-tariff measures as both converge in international trade. To begin, we analyze the tariff measure definition, the concept of non-tariff measures, as defined by the WTO, its main characteristics, effects and uses in national and international trade within the multilateral system.

KEYWORDS

Tariff, Regulatory Measures, International Trade, Non-Tariff Measures.

\footnotetext{
* Profesora en la Facultad de Derecho y Ciencias Sociales de la Benemérita Universidad Autónoma de Puebla, México (nataliagasparperez@gmail.com)
} 

das Arancelarias y Medidas No Arancelarias / 4. Efectos de las Medidas No Arancelarias

\section{Historia del arancel en México}

En México, la historia del arancel se remonta a épocas prehispánicas, ya que existía una amplia actividad comercial entre los pueblos mesoamericanos; que utilizaban el trueque, los sistemas de valor y medidas que se empleaban para el intercambio de mercancía. Las culturas dominantes exigían a los pueblos pequeños el pago de tributos e imponían una organización del comercio a grandes distancias, que resultó de gran importancia para la sociedad. ${ }^{1}$

La formalización del comercio en España se intensificó con la expedición de territorios y las colonias comenzaron a expedir las cédulas reales que certificaban el monopolio mercantil con los territorios recientemente conquistados. Ya en el virreinato (1521-1821) se estableció el derecho de almojarifazgo, impuesto a la importación y que permitía la entrada y salida de mercancías e incluso se hablaba de franquicias diplomáticas por la introducción de mercancías.

En la época de 1700 a 1800 el tráfico comercial se llevaba mediante esporádicas ordenanzas y cédulas reales. En 1728 se expidió una cédula a fin de que ninguna mercancía que ingresara por el puerto de Veracruz pudiera bajar a tierra sin que hubiera un consentimiento del oficial de justicia; en 1795 fue constituido el Consulado de Comerciantes de Veracruz, la primera asociación gremial cuyos miembros colaboraron en el mejoramiento del puerto y la ciudad.

Del año de 1800 a 1900 se expidió el primer documento legal del México independiente, llamado Arancel General Interno para los Gobiernos de las Aduanas Marítimas en el Comercio Libre del Imperio, en el cual se habilitaron los puertos para el comercio y se plantearon las bases para la operación del arancel, estableciendo los géneros y las mercancías de importación prohibida; las libres de gravamen quedaban a criterio de los administradores de las aduanas.

En 1951 se publicó el Código Aduanero, vigente hasta 1982, que señalaba los lugares para realizar la introducción o extracciones de mercancías. Poco después, la Ley Aduanera publicada en diciembre de 1981, que contenía la

1 "El Comercio Exterior A través de la Historia de México". [Consulta: 25 de junio de 2013] Disponible en: http://catarina.udlap.mx/u_dl_a/tales/documentos/lri/navarrete_m_e/capitulo2.pdf 
terminología utilizada internacionalmente, simplificó la estructura de los recursos administrativos haciendo referencia a los previstos en el Código Fiscal de la Federación que se regía por un principio de confianza al contribuyente a través de la autodeterminación del impuesto.

A partir de 1997, año en que se creó el Servicio de Administración Tributaria (SAT), se reformó la ley aduanera para revisar y fortalecer los mecanismos de control que permitieran revisar y fortalecer los mecanismos encaminados a combatir la evasión del pago de contribuciones. ${ }^{2}$

A lo largo de la historia, nuestro país ha tratado de acoplarse al comercio exterior para beneficios del mismo. En la actualidad se está dando una expansión de las relaciones comerciales a nivel mundial. A través de sus regímenes comerciales y cambios en las políticas económicas.

\section{ARANCEL}

Antes de abordar una definición técnica de un arancel, debemos tener en cuenta la finalidad o el uso que estos tienen en las relaciones comerciales de un país.

Los aranceles son utilizados por los gobiernos desde dos puntos específicos. Por un lado, para el control y protección de los mercados del mismo país en la importación de mercaderías; por el otro, se utilizan como protección de los productos fabricados en el país o de aquellos que son enviados al extranjero a través de los programas de fomento a la exportación. ${ }^{3}$

La Ley de Comercio Exterior nos da una definición de los aranceles: "Son las cuotas de las tarifas de los impuestos generales de exportación e importación".

Así como podemos definir a los aranceles desde un punto de vista jurídico o legal, también lo podemos hacer desde el punto de vista de los economistas. Tenemos diferentes definiciones:

- Derecho de aduana que grava las mercancías importadas por un país y que constituye el instrumento por excelencia del proteccionismo, favoreciendo a los productores nacionales. El arancel aduanero en algunas ocasiones se aplica también a las exportaciones de un país por meras necesidades económicas aumentando la recaudación tributaria.

\footnotetext{
${ }^{2}$ Cruz Barney, Oscar, "El Régimen Jurídico del Comercio Exterior de México: De la Independencia al Tratado de Libre Comercio de América del Norte", Universidad Nacional Autónoma de México. [Consulta: 25 de junio de 2013] Disponible en: http://www.juridicas.unam.mx/publica/librev/rev/ hisder/cont/18/pr/pr10.pdf

${ }^{3}$ Cátedra de la maestra Marta Elena Vázquez Vallejo. Régimen del Comercio Internacional. Facultad de Derecho y Giencias Sociales. Benemérita Universidad Autónoma de Puebla. Primavera 2012.
} 
- La tarifa oficial donde se recogen los aranceles aplicables a la entrada, salida y tránsito internacional de mercancías se denomina arancel de aduanas.

- Tarifa de impuesto que grava la importación o exportación de bienes y servicios. ${ }^{4}$

También encontramos al arancel en la contabilidad, definido como aquel impuesto que pagan los bienes que son importados a un país. De igual manera se puede definir como: aquellos "impuestos que se les agrega al precio normal de la mercancía, cuando esta es vendida, transportada o distribuida a empresas o comerciantes; y su precio es estipulado de acuerdo con el tipo de producto que se está comercializando".5

Interpretando lo descrito por ley y por los diferentes conceptos citados, y considerando diferentes materias en los que son utilizados y conocidos, podemos entender que los aranceles son aquellos impuestos que se cobran a los productos extranjeros para la protección de los productos nacionales, con el fin de que no surjan las llamadas prácticas desleales del comercio.

La ley de comercio divide los aranceles en tres tipos que el Estado aplica a las personas que exportan e importan mercancías. Estos se dividen de la siguiente manera: ad valorem; específicos y mixtos.

Los aranceles ad valorem son aquellos que se imponen en términos de porcentaje sobre el valor de las mercancías. Por ejemplo, 5\% de arancel significa que el arancel de importación es 5\% del valor de la mercancía en cuestión.

Los aranceles específicos son aquellos que se imponen en términos de cargas o cobros monetarios, específicos, por unidad o cantidad de mercancía importada. Por ejemplo, $\$ 100$ por tonelada métrica de la mercancía.

El arancel mixto es básicamente la combinación del arancel ad valorem y el específico. ${ }^{6}$ Los aranceles a que se hace referencia la ley, podrán adoptar las siguientes modalidades: Arancel-cupo y arancel estacional. El primero de ellos está enfocado para cuando se establezca una cuota arancelaria para cierta cantidad de mercancías a exportar o importar y una tasa diferente a las mercancías que excedan dicho monto establecido; el segundo se establecerá cuando los niveles arancelarios cambien dependiendo del periodo del año.

\footnotetext{
${ }^{4}$ La Gran Enciclopedia de Economía. Web informativa, 2006-2009. [Consulta: 15 de junio de 2013] Disponible en: http://www.economia48.com/spa/d/arancel/arancel.htm

5 "Aranceles: Una salvaguardia para la industria textilera de Colombia", 10 de Septiembre 2008. [Consulta: 15 de junio de 2013] Disponible en: http://www.gerencie.com/aranceles-una-salvaguardia-parala-industria-textilera-de-colombia.html

${ }^{6}$ Guía de Arancel. [Consulta: 22 de mayo de 2013]. Disponible en: http://www.camaras.org/guias/arancel/guia_arancel_cap2_020.html
} 
También nos menciona la ley que podrán establecerse aranceles diferentes a los generales de exportación e importación cuando así lo establezcan tratados o convenios comerciales internacionales de los que México sea parte.

Es entonces que podemos definir al arancel, como aquella terminología oficial de las mercancías clasificadas con la determinación de los gravámenes que deben de producirse con la importación o exportación. Establecer un arancel tiene tres consecuencias principales: en primer lugar, anima a las empresas a producir; en segundo lugar, induce a los consumidores a comprar productos nacionales y en un mínimo, consumir los productos que llegan al país con aranceles altos; y, finalmente, eleva los ingresos del Estado

$\mathrm{Al}$ decir o hablar de terminología nos referimos básicamente a la clasificación que tienen los aranceles. Esta clasificación arancelaria consiste en la ubicación de una determinada mercancía en la fracción que le corresponde dentro de la Tarifa de los Impuestos Generales de Importación y Exportación y, se realiza aplicando las Notas de Sección, las Notas de Capítulo, las Reglas Complementarias y las Notas Explicativas de la referida tarifa, así como de diversos criterios que existen sobre clasificación. ${ }^{7}$

Los responsables de realizar tal clasificación son los agentes aduanales o los apoderados aduanales con la información proporcionada por quien contrate el servicio para que así sepa cuánto van a pagar y cumplan con las regulaciones y restricciones no arancelarias.

Tomemos como ejemplo de clasificación arancelaria el de los teléfonos celulares. Basados en la Tarifa de la Ley de Impuestos Generales de Importación y Exportación (LIGIE), las fracciones arancelarias se componen de: Capítulo, partida, subpartida y fracción.

1. Capítulo: 85, que se refiere a Máquinas, aparatos y material eléctrico, y sus partes; aparatos de grabación o reproducción de sonido, aparatos de grabación o reproducción de imagen y sonido en televisión, y las partes y accesorios de estos aparatos.

2. Partida: 8517 que se refiere a teléfonos, incluidos los teléfonos celulares y los de otras redes inalámbricas; los demás aparatos de transmisión o recepción de voz, imagen u otros datos, incluidos los de comunicación en red con o sin cable (tales como redes locales - LAN- o extendidas -WLAN-), distintos de los aparatos de transmisión o recepción de las partidas 84.43, 85.25, 85.27 u 85.28.

3. Subpartida: 851712, que se refiere a teléfonos celulares y los de otras redes inalámbricas.

\footnotetext{
7 "Clasificación y Valor. Aduana México Servicio de Administración Tributaria". Abril de 2006. [Consulta: 15 de junio de 2013] Disponible en: http://www.aduanas.sat.gob.mx/aduana_mexico/Descargas/Guia_Importacion/GI06_06.pdf
} 
1. Fracción Arancelaria: 85171201 que se refiere a aparatos emisores con dispositivo receptor incorporado, móviles, con frecuencias de operación de 824 a $849 \mathrm{MHz}$ pareado con 869 a $894 \mathrm{MHz}$, de 1,850 a 1,910 MHz pareado con 1,930 a 1,990 MHz, de 890 a $960 \mathrm{MHz}$ o de 1,710 a 1,880 MHz, para radiotelefonía (conocidos como "teléfonos celulares”). Las Restricciones para esta fracción arancelaria son:

Fracción arancelaria 85171201 se encuentra sujeta a lo siguiente:

I. Aranceles

A la importación: exento del Impuesto General de Importación.

A la exportación: exento del Impuesto General de Importación.

A la importación: 16\% de IVA

A la exportación: exento de IVA

Unidad de medida: pieza

II. Regulaciones y restricciones no arancelarias.

A la Importación: Norma oficial Mexicana (Información Comercial) de la NOM-024-SCFI-1998 (El importador podrá optar por cualquiera de las alternativas previstas en el Art. 6 para comprobar el cumplimiento de la NOM)

III. Anexos. No aplica

IV. Cupos: No aplica ${ }^{8}$

Con este ejemplo tratamos de revelar la importancia de esta clasificación en el comercio exterior, ya que al momento de clasificarlas se cumple con los requerimientos legales estipulados por la ley para así poder ingresar las mercancías de comercio internacional al territorio nacional.

De todo lo abordado, tenemos que el establecimiento de un arancel es benéfico en cuanto al comercio estratégico, la protección de las industrias nacionales, el desarrollo económico nacional y la protección contra las prácticas desleales del comercio exterior.

\section{Medidas arancelarias y medidas no arancelarias}

Una vez que hemos entendido qué es un arancel hablaremos de sus medidas de regulación. Las medidas no arancelarias están consideradas como un reto para el sistema multilateral de comercio, por lo que al utilizarlas se busca que no provoquen una distorsión en el comercio pero, al mismo tiempo, poder recurrir a ellas para alcanzar objetivos políticos necesarios para el comercio exterior. ${ }^{9}$

${ }^{8}$ Ejemplo de una clasificación arancelaria. Vermarkten: Planeación y Desarrollo Integral en Comercio Exterior. 2011. [Consulta: 15 de junio de 2013] Disponible en: http://www.vermarkten.com.mx/importaciones/ejemplo-de-una-clasificacion-arancelaria-.html

${ }_{9}^{9}$ Arcos Reinah, Samantha Esther y Santillán Veja, Patricia, "Aranceles y Medidas de Regulación y 
En los primeros años de existencia del GATT, ${ }^{10}$ las principales medidas en las que se concentraba la atención eran aquellas que se relacionaban con la balanza de pagos, el empleo y las relaciones encaminadas al desarrollo. Sin embargo, en la actualidad la atención está viéndose encaminada a aquellas medidas que están relacionadas con preocupaciones técnicas, sanitarias y ambientales. La expansión de las normas comerciales a nuevas esferas ha provocado que la discusión de la utilización de estas medidas no arancelarias se vuelva aún más compleja e incluso genere tensión entre las normas internacionales y la soberanía en materia de políticas. ${ }^{11}$

Desde la apertura del comercio, el GATT (Organización Mundial de Comercio - $\mathrm{OMC}-$ desde 1994) estableció una distinción normativa básica entre las medidas arancelarias y no arancelarias; en particular, favoreció el recurso a los aranceles, ya que estos se consideraban una forma "equitativa de protección” en las consecuencias económicas y más susceptibles de ser objeto de reducciones mediante negociaciones. Las restricciones cuantitativas y otras medidas no arancelarias se consideraban discriminatorias y perturbadoras de las fuerzas del mercado.

En la historia de las medidas no arancelarias, la diversidad de formas que adoptaban y el hecho de que en muchos casos la finalidad política de las medidas solo guardaba una relación indirecta con el comercio, los arquitectos del GATT no lograron definir un enfoque global que abarcara todas las medidas no arancelarias y trataron las distintas clases de medidas de forma diferente.

Las restricciones cuantitativas eran las medidas no arancelarias más importantes en el momento en que se estaba redactando el GATT, de manera que no es de sorprenderse que sean objeto de disposiciones detalladas y complejas. Las disposiciones del GATT regularon otras medidas no arancelarias, sin prohibirlas, para garantizar que las necesarias y legítimas políticas internas no fueran discriminatorias y entrañaron el menor grado de restricción del comercio posible. ${ }^{12}$

\footnotetext{
Restricción no Arancelaria al Comercio Exterior", Universidad Autónoma del Estado de Hidalgo, Instituto de Ciencias Económico Administrativas, Monografía Profesional. Pachuca, Hidalgo, septiembre 2005. [Consulta: 26 de junio de 2013] Disponible en: http://www.uaeh.edu.mx/docencia/Tesis/icea/ licenciatura/documentos/Aranceles $\% 20 \mathrm{y} \% 20$ medidas.pdf4ezb

${ }^{10}$ General Agreement on Tariffs and Trade/ Acuerdo General sobre Aranceles y Comercio (GATT 1947). "Acuerdo de igualdad de trato y multilateral que regula el comportamiento a seguir por los estados firmantes en la práctica del comercio internacional". [Consulta: 15 de junio de 2013] Disponible en: http://biblio.juridicas.unam.mx/libros/1/377/24.pdf

${ }^{11}$ Alvares Soberanis, Jaime, "El GATT: Antecedentes y Propósitos". Universidad Iberoamericana. UNAM. [Consulta: 26 de junio de 2013] Disponible en: http://www.juridicas.unam.mx/publica/librev/rev/jurid/cont/11/pr/pr5.pdf

${ }^{12}$ Informe sobre el Comercio Mundial 2012. Comercio y Políticas Publicas: análisis de las medidas no arancelarias en el Siglo XXI, Organización Mundial del Comercio, pp. 94-125. [Consulta: 26 de junio de 2013] Disponible: http://www.wto.org/spanish/res_s/booksp_s/anrep_s/world_trade_report12_s.pdf
} 
La evolución de los temas centrales y el alcance de las rondas de negociaciones del GATT no solo ponen de manifiesto la importancia permanente de las medidas no arancelarias para el sistema internacional de comercio, sino también que la importancia relativa de las distintas medidas ha cambiado con el tiempo. Las restricciones cuantitativas eran el problema al que tuvieron que hacer frente los primeros negociadores del GATT.

La expansión del comercio mundial, la profundización de la integración de las economías y la ampliación y fortalecimiento de las normas comerciales han conducido inevitablemente a que las medidas no arancelarias sean un elemento cada vez más destacado en el panorama del comercio internacional. La reducción de la protección arancelaria ha llevado a algunos países a recurrir en mayor medida y de forma más creativa a la aplicación de medidas no arancelarias, ${ }^{13}$ las cuales constituyen medios de regulación al comercio exterior, que tienen por objeto la protección de una serie de situaciones que guardan precisa relación con la economía del país. Todas estas medidas de control y regulación deben ser sometidas a la aprobación de la Comisión de Comercio Exterior ${ }^{14}$ y publicarse en el Diario Oficial de la Federación (DOF) con base en las reglas que establezca la Ley de Comercio Exterior.

También nos establece que las autoridades competentes (SAGARPA, SEMARnat, SEDEna, Secretaría de Salud) podrán dictar estas medidas sin la aprobación previa de dicha comisión, siempre que estas se publiquen en el DOF y tengan una duración máxima de veinte días. En su artículo 15, la ley menciona los casos en los que se podrán establecer dichas medidas.

- Las medidas de regulación, expedidas por la Secretaria de Economía (SE), se podrán establecer en caso de protección de materias primas y productos destinados al consumo básico. De igual manera, para la protección de los recursos naturales no renovables, la fauna y flora en riesgo para asegurar su conservación; también para el resguardo de bienes de valor histórico y aquellas situaciones que hagan referencia a medidas de seguridad, salud pública, ecología, que no estén reguladas por las Normas Oficiales Mexicanas.

- Las medidas que sean expedidas tanto por la Secretaría de Economía como por la autoridad competente se aplicarán en casos que requieran de corrección en cuanto a los pagos; cuando se trate de

${ }^{14}$ De acuerdo con el portal de la Asociación Nacional de la Industria Química (ANIQ), "La Comisión de Comercio Exterior es un grupo de trabajo coordinado por la Dirección de Información y Comercio Exterior en el cual participan las empresas afiliadas y tiene como función principal la vigilancia en el cumplimiento del plan de trabajo, así como la de aprobar las posturas de la Asociación en los diferentes temas en que interviene". [Consulta: 16 de abril de 2013]. Disponible en: http://www. aniq.org. $\mathrm{mx} /$ comisiones/ccomercio.asp
} 
regular la entrada de productos usados; como medio de respuesta a restricciones de exportación a mercancías mexicanas y cuando se requiera impedir la entrada de mercancías que generen prácticas desleales de comercio.

De acuerdo a la Ley de Comercio Exterior, las restricciones y regulaciones no arancelarias deberán cumplirse conforme a lo que se establece en los ordenamientos aplicables. Estos cumplimientos deberán demostrarse mediante documentos que contengan medidas de seguridad o a través de medios electrónicos o ambos que sean determinados por la Secretaría de Economía. Dichos documentos deberán acompañar al pedimento que sea presentado en la aduana en los términos de la legislación aduanera.

Para establecer las regulaciones no arancelarias, el artículo 20 de la Ley de Comercio Exterior nos dice que se aplicaran de acuerdo a su fracción y nomenclatura arancelaria dependiendo de la tarifa que corresponda a estas. ${ }^{15}$

Para que estas medidas se cumplan la Ley de Aduanera, en su artículo 36 , refiere que todas aquellas personas que importen o exporten mercancías deberán presentar un pedimento en la forma oficial aprobada por la Secretaría de Economía, por medio de apoderado o agente aduanal.

Dicho pedimento tendrá especificaciones especiales para cada una de las actividades a realizar. En caso de importación, el pedimento deberá de ser acompañado de:

1. La factura que reúna los datos que establezca la Secretaría cuando el valor de las mercancías, en la aduana, se determine en cuanto a su valor de transacción y este rebase la cantidad establecida por tales reglas.

2. El conocimiento de embarque.

3. Los documentos que demuestren el cumplimiento de dichas regulaciones, que hayan sido expedidas conforme a lo establecido por la Ley de Comercio Exterior, siempre y cuando sean publicadas estas en el DOF y sean identificados en cuanto a la fracción y nomenclatura arancelaria.

4. Documento que determine la procedencia y origen de las mercancías en cuestión.

5. Documento de la garantía otorgada cuando el valor de la mercancía cuando el valor declarado sea menor.

6. Certificado de peso o volumen

\footnotetext{
${ }^{15}$ Secretaría de Economía. "Manual de Regulaciones No Arancelarias", agosto 2011, p. 7. [Consulta: 23 de mayo de 2013]. Disponible en: http://www.economia.gob.mx/files/comunidad_negocios/industria_comercio/RegulacionesnoArancelarias2011.pdf
} 
En la exportación:

1. Factura que determine el valor de las mercancías

2. Los documentos que demuestren el cumplimiento de dichas regulaciones, que hayan sido expedidas conforme a lo establecido por la Ley de Comercio Exterior, siempre y cuando sean publicadas estas en el DOF, y sean identificados en cuando a la fracción y nomenclatura arancelaria.

En este caso, el Sistema de Administración Tributaria (SAT) podrá requerir que al pedimento se le acompañen los documentos aduaneros que sean necesarios, conforme a los acuerdos internacionales reconocidos por México.

A los documentos que deban ser presentados con las mercancías, el SAT podrá señalar obligaciones que podrán ser cumplidas de manera electrónica o por envío en forma digital. En materia de sanidad animal y vegetal, la misma deberá de ser revisada en el área de fiscalización establecida en las aduanas que señale el sAT.

Tomando el mismo ejemplo de medidas arancelarias de los celulares, en la Norma Oficial Mexicana que se maneja en el ejemplo, tenemos que en medidas y restricciones no arancelarias, las normas o especificaciones de ese producto serán:

los productos eléctricos, electrónicos y electrodomésticos, deben ir acompañados o tener impresos sobre el empaque, los instructivos y advertencias para su uso normal, conservación y mejor aprovechamiento, así como las advertencias para el manejo seguro y confiable de los mismos [...] Cuando se trate de productos electrónicos, equipos de procesamiento de datos, de informática y accesorios, podrán cumplir con lo indicado en lo anteriormente señalado, o bien ir acompañados de instructivos; estos instructivos, con la información detallada para su uso normal, y mejor aprovechamiento de los mismos, podrán proporcionarse a través de medios de almacenamiento e incluso, direcciones de internet $[\ldots]$ Los instructivos y advertencias deben redactarse en idioma español y en términos comprensibles y legibles, sin perjuicio de que además se expresen en otros idiomas [...] Los fabricantes, nacionales o importadores de productores, que pudieran resultar peligrosos, tienen la obligación de informar las advertencias necesarias de forma clara y manifiesta para el manejo seguro de los mismos; para efectos de esta norma, los equipos, altamente especializados que no se detallen directamente y cuya comercialización no sea el uso doméstico, si no para uso especiales, no requieren instructivos, ni de advertencias por ser instalados por el personal técnico especializado del proveedor ${ }^{16}$

${ }^{16}$ Norma Oficial Mexicana. Información Comercial para empaques, instructivos y garantías de los 
Con este ejemplo demostramos cómo convergen las medidas arancelarias y no arancelarias traducidas en especificaciones técnicas que cada país establece, en uso de su soberanía, sin contraponerse a los acuerdos multilaterales.

\section{EFECTOS DE LAS MEDIDAS NO ARANCELARIAS}

Las medidas no arancelarias son aquellas medidas impuestas sobre los flujos de comercio que no sea una medida arancelaria. Algunas de estas medidas pueden constituirse en barreras no arancelarias. ${ }^{17}$

La Organización Mundial del Comercio permite de cierta manera a los gobiernos el uso de medidas que pueden afectar al comercio para proteger sus intereses nacionales, siempre y cuando no sean discriminatorias o constituyan prácticas desleales en el comercio. La mayor parte de estas medidas son utilizadas en cuanto a salud, medidas fitosanitarias, la protección de la vida humana y la preservación de sus animales y vegetales. ${ }^{18}$

Las medidas no arancelarias difieren de los aranceles en varios aspectos. Estas diferencias, unidas a la naturaleza cambiante del comercio internacional, pueden constituir razones adicionales para la cooperación en materia de medidas no arancelarias en el marco de acuerdos comerciales. Las medidas no arancelarias responden con frecuencia a preocupaciones cruciales de política pública a nivel nacional e internacional. Su objeto puede ser proteger los intereses generales de los consumidores más que las limitadas preocupaciones de los productores. La preservación de los vegetales, la protección de la salud de las personas y de los animales, la seguridad de los alimentos y el medio ambiente o el establecimiento de las normas necesarias para un intercambio equitativo en el mercado son objetivos de política pública. Estos objetivos, aunque son ampliamente compartidos por los miembros de la OMC, presentan a menudo una amplia visión de preferencias puras en cuanto a las políticas. Además, las medidas no arancelarias y los aranceles son diferentes en lo que respecta a su duración. Las medidas no arancelarias están sujetas a modificación porque las necesidades de reglamentación varían de acuerdo con las transformaciones del entorno económico y social. ${ }^{19}$

\footnotetext{
productos electrónicos, eléctricos y electrodomésticos. Secretaría de Comercio y Fomento Industrial. [Consulta: 29 de junio de 2013] Disponible en: http://www.aduanas-mexico.com.mx/claa/ctar/normas/nm024bsc.htm

17 "Sistema de información de Comercio Exterior", Diccionario de Términos de Comercio, 2013. [Consulta: 23 de mayo de 2013]. Disponible en: http://www.sice.oas.org/dictionary/TNTM_s.asp

${ }^{18}$ Organización Mundial del Comercio, "La Facilitación del Comercio", [Consulta: 23 de mayo de 2013] Disponible en:http://www.wto.org/spanish/tratop_s/tradfa_s/tradfa_s.htm

${ }^{19}$ Informe sobre el Comercio Mundial 2012. Comercio y Políticas Publicas: análisis de las medidas no arancelarias en el Siglo XXI, Organización Mundial del Comercio, pp. 134-152. [Consulta: 26 de junio de 2013] Disponible: http://www.wto.org/spanish/res_s/booksp_s/anrep_s/world_trade_report12_s.pdf
} 
Las medidas sanitarias y fitosanitarias son de igual importancia para los productores, los comerciantes y los consumidores. Una de sus cuestiones primordiales es como abordar los efectos desfavorables de las medidas no arancelarias en el comercio, sin llegar a ser una traba para los gobiernos en la búsqueda de alcanzar los objetivos de política pública como la protección a la salud.

La naturaleza cambiante del comercio internacional hace que surjan nuevas consideraciones de política que pueden crear la necesidad de formas de integración institucional más profundas. De igual manera, la creciente preocupación por las medidas sanitarias y fitosanitarias ha hecho que se plantee en la oma la convergencia normativa. El camino a la afinidad no siempre es fácil, ya que no se trata de cuestiones mecánicas de formulación de políticas y entren en un juego de diferencias nacionales en cuanto a las preferencias y prioridades sociales.

México es miembro de la Organización Mundial del Comercio desde el 1 de enero de 1995, y de este se desprende un Acuerdo sobre Obstáculos Técnicos al Comercio, el cual pretende garantizar que los reglamentos y normas establecidas, así como los procedimientos de certificación, no generen ningún tipo de obstáculos al comercio internacional. ${ }^{20}$ Este Acuerdo es directo en su objetivo, pero al mismo tiempo que busca la no obstaculización al comercio, intenta apoyar la colaboración entre los miembros destacando el apoyo a los países miembros en desarrollo en los esfuerzos que realicen dentro de esta esfera.

En virtud del acuerdo, se establecerá un comité de Obstáculos Técnicos al Comercio, que será constituido por representantes de los países miembros y cuya principal función será vigilar el funcionamiento de dicho acuerdo y establecer grupos de trabajo para desarrollar las funciones que les sean asignadas con base en el propio acuerdo, sin necesidad de duplicar estas.

Además de lo establecido por la Organización Mundial del Comercio, en lo referente a medidas no arancelarias, la regulación de estas se señala en los Tratados y Acuerdos de Libre Comercio de los que México es parte, de los cuales solo mencionaré los que han entrado en vigor en los años:

\footnotetext{
${ }^{20}$ Organización Mundial del Comercio, "Acuerdo sobre Obstáculos Técnicos al Comercio", Textos Furídicos, 1994 [Consulta: 23 de mayo de 2013] Disponible en: http://www.wto.org/spanish/docs_s/ legal_s/17-tbt_s.htm
} 


\begin{tabular}{|c|c|c|c|}
\hline Tratado & $\begin{array}{l}\text { Entrada } \\
\text { en vigor }\end{array}$ & $\begin{array}{l}\text { Medidas no } \\
\text { arancelarias }\end{array}$ & Objetivo \\
\hline $\begin{array}{l}\text { Tratado de } \\
\text { Libre Comercio } \\
\text { México-Cen- } \\
\text { troamerica }\end{array}$ & 2012-2013 & $\begin{array}{l}\text { Capítulo IX } \\
\text { Obstáculos Técni- } \\
\text { cos al Comercio. }\end{array}$ & $\begin{array}{l}\text { Facilitar e incrementar el comer- } \\
\text { cio de mercancías, identificando, } \\
\text { previendo y eliminando obstá- } \\
\text { culos incensarios, que puedan } \\
\text { surgir como consecuencia de la } \\
\text { preparación, adopción, y apli- } \\
\text { cación de normas, reglamentos } \\
\text { técnicos y procedimientos. }\end{array}$ \\
\hline $\begin{array}{l}\text { Acuerdo de Inte- } \\
\text { gración Comer- } \\
\text { cial México-Perú }\end{array}$ & Febrero 2012 & $\begin{array}{l}\text { Capítulo VIII } \\
\text { Obstáculos Técni- } \\
\text { cos al Comercio }\end{array}$ & $\begin{array}{l}\text { Incrementar y facilitar el comer- } \\
\text { cio bilateral evitando que las } \\
\text { normas, reglamentos técnicos y } \\
\text { procedimientos de evaluación } \\
\text { de la conformidad constitu- } \\
\text { yan obstáculos innecesarios al } \\
\text { comercio, así como aumentar la } \\
\text { cooperación y asistencia técnica } \\
\text { entre las Partes. }\end{array}$ \\
\hline
\end{tabular}

En México, además del acuerdo anteriormente citado, se cuenta con otra normatividad, aplicada a las medidas tomadas para la importación de mercancías, entre ellas permisos previos, cupos y Normas Oficiales Mexicanas (NOM).

Solo como ejemplo para entender este tipo de normatividad y las medidas arancelarias analizaremos, de manera muy técnica y práctica, el Tratado de Libre Comercio entre México y Bolivia de 1995, que a pesar de tener ya varios años de vigencia es una muestra de la aplicación de dichas medidas en el comercio internacional. El Tratado de Libre Comercio entre México y Bolivia del 11 de enero de 1995 tiene el carácter de Acuerdo de Complementación Económica. Su objetivo consiste en establecer una Zona de Libre Comercio con reglas específicas que beneficien a los países miembros en materia de comercio e inversión. Las medidas aplicadas en este tratado buscan la expansión y diversificación del comercio con nuestro país. El tratado comprende medidas arancelarias que permiten la regulación y la práctica comercial entre ellos y con los países que deseen importar mercancías sin que se encuentren adscritos a este tratado. Se buscaba que cuando se adoptaran restricciones a importaciones de un país que no fuera miembro, debería ser consultado el tratado por estas para evitar que tales medidas causaran o fueran partícipes de distensiones en los precios de comercialización y distribución.

De manera general, entendemos que las medidas no arancelarias son aquellas utilizadas para regular y controlar las operaciones de comercio exterior y, de esta manera, proteger la economía nacional y verificar que las actividades comerciales realizadas sean lícitas y no caer en las prácticas 
desleales en el comercio, para evitar los daños a los mercados, en conjunto con apoyo de leyes, convenios y regulaciones que respalden la seguridad y buen manejo de las prácticas comerciales.

Cabe destacar que en los nuevos escenarios de integración económica latinoamericana, como el MERcosur y la Comunidad Andina, sobresalen interesantes esquemas de medidas no arancelarias, no solo como una protección del mercado, sino del propio derecho del consumidor, como es el caso de Brasil, donde desde hace 20 años se tiene una cultura de Protección y Defensa del Consumidor. ${ }^{21}$ Ante esto hay que reflexionar si estas medidas arancelarias y no arancelarias nos garantizan una protección como consumidores. Se queda solo como reflexión de lo que ya debemos empezar a trabajar para la creación de políticas públicas de la mano con órganos reguladores de protección al consumidor.

\footnotetext{
${ }^{21}$ Pilau Sobrinho, Liton Lanes y Da Silva, Rogerio (org.), Balcâo do consumidor, 20 anos do Código de Defensa do consumidor, Brasil, Passo Fundo, UPF, 2011.
} 
